\title{
Focusing light into biological tissue: how effective is it?
}

\section{Lihong V. Wang}

Lihong V. Wang, "Focusing light into biological tissue: how effective is it?," Proc. SPIE 3914, Laser-Tissue Interaction XI: Photochemical, Photothermal, and Photomechanical, (13 June 2000); doi: 10.1117/12.388053 


\title{
Focusing light into biological tissue - How effective is it?
}

\author{
Lihong V. Wang \\ Optical Imaging Laboratory, Biomedical Engineering Program \\ Texas A\&M University, College Station, Texas 77843-3120 \\ URL: http://oilab.tamu.edu; Email: LWang@tamu.edu
}

\begin{abstract}
Focusing light into a turbid medium was studied with Monte Carlo simulations. Focusing was found to have a significant impact on the absorption distribution in turbid media when the depth of the focal point (the distance between the focal point and the surface of the turbid media) was less than or comparable with the transport mean free path. Focusing could significantly increase the peak absorption and narrow the absorption distribution. As the depth of the focal point increased, the peak absorption decreased, and the depth of peak absorption increased initially but quickly reached a plateau that was less than the transport mean free path. A refractive-index-mismatched boundary between the ambient medium and the turbid medium deteriorated the focusing effect, increased the absorption near the boundary, lowered the peak absorption, and broadened the absorption distribution.
\end{abstract}

\section{INTRODUCTION}

The study of applications of laser and incoherent light in biomedicine for both therapeutic and diagnostic purposes is an active research field. It is possible to treat lesions selectively without damaging the surrounding normal tissues by use of selective interactions between light and biological tissue. The selectivity, related to one or more of the characteristics of laser light, includes the wavelength selectivity of light absorption by certain tissue types where the monochromatic property of laser light is employed or the thermal or stress confinement selectivity where the short pulse width of laser light is used. It is also possible to diagnose disease noninvasively by use of the optical properties of biological tissues. The optical properties include index of refraction, absorption coefficient, scattering coefficient, and scattering anisotropy. The spectra of the optical properties are related to the molecular conformation of biological tissues and hence should be sensitive indicators of the physiological status of biological tissues.

One of the fundamental goals in the study of laser-tissue interaction is to understand and simulate light transport in optically turbid biological tissues, by use of various theoretical models. In these models the optical properties of biological tissues are the input parameters, and the light distribution in the tissues or re-emitted from the tissues is calculated. Several methods are usually used in the modeling. The first method starts from the radiative transfer equation. The radiative transfer equation is usually too complex to solve analytically and is often simplified by a diffusion approximation. The radiative transfer equation with the approximation is usually solved by finite-element or finite-difference methods. ${ }^{1,2}$ The second method is based on the Monte Carlo technique. Other methods include discrete random-walk model ${ }^{3}$ and path-integral technique. ${ }^{4}$ Diffusion theory is quick but inaccurate in predicting the light distribution near the light source and boundaries. In many applications, especially the therapeutic applications, knowledge about the light distribution near the source is very important. On the contrary, the Monte Carlo method is accurate but slow owing to its statistical nature.

In this work, we studied light distribution in turbid media when a focused optical beam was used to enhance the peak absorption inside the media. Monte Carlo technique was employed for its accuracy and flexibility in dealing with complex configurations. Although focusing optical beams has been well studied in confocal microscopy for imaging applications, ${ }^{5}$ our goal was to investigate the internal light absorption toward therapeutic applications. 


\section{METHOD}

Monte Carlo simulations of light transport in tissues have been implemented previously for simple tissue geometry. ${ }^{6-13}$ To compute light distributions according to the tissue geometry and optical properties, including refractive index $n$, absorption coefficient $\mu_{a}$, scattering coefficient $\mu_{s}$, and anisotropy factor $g$, we have written a Monte Carlo program in $C$ for tissues with buried objects and focused optical beams. We used the delta-scattering technique ${ }^{14}$ for photon tracing to greatly simplify the algorithm because this technique allows a photon packet to be traced without directly dealing with photon crossings of interfaces between different types of tissues.

A Cartesian coordinate system was set up for the simulation. The origin of the coordinate system was the center of the incident optical beam on the surface of the turbid medium. The $z$ axis was the normal of the surface pointing toward the inside of the turbid medium. The $x y$ plane was therefore on the surface of the turbid medium.

For a focused optical beam of a radius $\rho_{f}$, the irradiance was assumed to be uniform on the surface of the turbid medium (Fig. 1), and the radial position of a photon packet was sampled by

$$
\rho=\rho_{f} \sqrt{x_{r}}
$$

where $\xi_{\rho}$ was a uniformly distributed random number between 0 and $1\left(0 \leq \xi_{\rho} \leq 1\right)$. The azimuthal angle of the photon packet was sampled by

$$
\theta=2 \pi \xi_{\theta},
$$

where $\xi_{\theta}$ was another uniformly distributed random number between 0 and $1\left(0 \leq \xi_{\theta}<1\right)$. The Cartesian coordinates of the incident point were

$$
\begin{aligned}
& x=\rho \cos (\theta), \\
& y=\rho \sin (\theta),
\end{aligned}
$$

If the depth of the focal point in the turbid medium was $z_{f}$, the directional cosines were set to

$$
\begin{gathered}
u_{x}=-x / \sqrt{r^{2}+z_{f}^{2}}, \\
u_{y}=-y / \sqrt{r^{2}+z_{f}^{2}}, \\
u_{z}=z_{f} / \sqrt{r^{2}+z_{f}^{2}},
\end{gathered}
$$

If the ambient medium and the turbid medium had the same index of refraction, the directional cosines did not need to be changed when the photon packet entered the turbid medium. Otherwise, the directional cosines were changed based on the Snell's law, and the specular reflection was taken into account based on the Fresnel equations. Once the photon packet was launched into the turbid medium, the photon packet was traced with the delta-scattering technique.

The tissue system in the simulation had multiple types of tissue such as epidermis and dermis, which were assumed to have the same index of refraction. The interaction coefficient (also known as the total attenuation coefficient) of the $i$ th tissue type, defined as the sum of $\mu_{a}$ and $\mu_{s}$, was denoted by $\mu_{i}$. The delta-scattering technique applied to light transport in turbid media is briefly summarized as follows.

1. Define an upper-bound interaction coefficient $\mu_{m}$, where $\mu_{m} \geq \mu_{i}$ for all $i$. In this study, $\mu_{m}$ was set to the maximum $\mu_{i}$ among all tissue types.

2. Select a step size $R$ between two consecutive interactions based on the upper-bound interaction coefficient,

$$
R=-\ln \left(\xi_{R}\right) / \mu_{m},
$$

where $\xi_{R}$ was a uniformly distributed random number between 0 and $1\left(0<\xi_{R} \leq 1\right)$. Then, determine the tentative next collision site $\boldsymbol{r}_{k}{ }^{\prime}$ by

$$
\boldsymbol{r}_{k}^{\prime}=\boldsymbol{r}_{k-1}+R \boldsymbol{u}_{k-1}
$$

where $\boldsymbol{r}_{k-1}$ was the current site, and $\boldsymbol{u}_{k-1}$ was the direction of the flight. The direction of the flight was determined according to the probability distributions of the deflection (polar) angle and azimuthal angle at each interaction site. ${ }^{10}$

3. Play a rejection game:

a. Get another random number $\xi_{a}$, which was uniformly distributed between 0 and $1\left(0<\xi_{a}\right.$ $\leq 1)$.

b. If $\xi_{a} \leq \mu_{i}\left(\boldsymbol{r}_{k}\right) / \mu_{m}$, i.e., with a probability of $\mu_{i}\left(\boldsymbol{r}_{k}\right) / \mu_{m}$, accept this point as a real interaction site $\left(r_{k}=r_{k}^{\prime}\right)$. 
c. Otherwise, do not accept $\boldsymbol{r}_{\boldsymbol{k}}{ }^{\prime}$ as a real interaction site but select a new path starting from $\boldsymbol{r}_{\boldsymbol{k}}{ }^{\prime}$ with the unchanged direction $\boldsymbol{u}_{k-1}$ (i.e., set $\boldsymbol{r}_{k-1}=\boldsymbol{r}_{k}{ }^{\prime}$ and return to Step 2).

At each real interaction site, a fraction $\mu_{a} /\left(\mu_{a}+\mu_{s}\right)$ of the photon packet was absorbed, and the rest was scattered. The tracing continued until either the weight of the photon packet was below a preset threshold or the packet exited the tissue system. The weight threshold was set to $10^{-4}$ in this study. A sub-threshold photon packet experienced a "Russian roulette" to conserve energy. Since step 3 was very efficient, the delta-scattering technique was comparable with the standard Monte Carlo simulation methods in computational speed. The detailed treatment of photon tracing after step 3 was similar to that in Ref. 10 and will not be repeated here.

During the tracing of each weighted photon, ${ }^{10}$ the light absorption, reflection, or transmission were correspondingly scored into different arrays according to the spatial positions of the photon. Multiple photons were traced to achieve an acceptable statistical variation. For this study, two to ten million photons were traced.

It is assumed in the delta-scattering technique that all the tissues in the simulation have the same index of refraction, although the ambient medium (e.g., air) is allowed to have different index of refraction from the tissues. Since most soft tissues have similar indices of refraction, this limitation does not pose a problem in our simulation.

This Monte Carlo program was used to simulate the optical absorption for the two types of configurations shown in Fig. 1. Figure 1(a) shows an optical beam focused into a homogeneous turbid medium. Figure 1(b) shows an optical beam focused into a blood vessel buried $0.015 \mathrm{~cm}$ deep in a skin model unless stated otherwise. The refractive index of the ambient medium was chosen to be either $n_{a}=1$ or $n_{a}=1.4$. In Fig. 1(a), the optical properties of the turbid medium were $n_{l}=1.4, \mu_{a}=0.1 \mathrm{~cm}^{-1}, \mu_{s}=100$ $\mathrm{cm}^{-1}$, and $\mathrm{g}=0.9$. In Fig. 1(b), the optical properties of the epidermis were $n_{t}=1.4, \mu_{a}=19 \mathrm{~cm}^{-1}, \mu_{s}=480$ $\mathrm{cm}^{-1}$, and $\mathrm{g}=0.787$. The optical properties of the dermis were $n_{t}=1.4, \mu_{a}=2.2 \mathrm{~cm}^{-1}, \mu_{s}=210 \mathrm{~cm}^{-1}$, and $\mathrm{g}$ $=0.787$. The optical properties of the blood vessel were $n_{t}=1.4, \mu_{a}=266 \mathrm{~cm}^{-1}, \mu_{s}=473 \mathrm{~cm}^{-1}$, and $\mathrm{g}=$ 0.995. These optical properties for the skin model represented the realistic optical properties of the skin components at the wavelength of $532 \mathrm{~nm} .^{15}$ The thickness of the epidermis was $0.006 \mathrm{~cm}(60 \mu \mathrm{m})$. The dermis was considered semi-infinite in this simulation. The blood vessel was modeled as an infinitely long cylinder perpendicular to the schematic with a diameter of $0.006 \mathrm{~cm}(60 \mu \mathrm{m})$. The distance between the center of the cylinder and the surface of the turbid medium was varied. When focusing was applied, the depth of the focal point always matched the depth of the center of the blood vessel.

\section{RESULTS AND CONCLUSIONS}

The distributions of the absorption density in the homogeneous turbid medium as shown in Fig. 1(a) were simulated. The absorption density was in the units of $\mathrm{cm}^{-3}$ representing the absorption probability density of the incident photons per unit volume. If the light source was continuous wave, the absorption density multiplied by the incident power yielded the power deposition density in $\mathrm{W} / \mathrm{cm}^{-3}$. If the light source was pulsed, the absorption density multiplied by the incident energy yielded the energy deposition density in $\mathrm{J} / \mathrm{cm}^{-3}$.

When the indices of refraction between the ambient medium and the turbid medium were matched, $n_{a}=n_{t}=1.4$. The depth of the focal point varied among $z_{f}=0.05,0.1,0.2$, and $\infty \mathrm{cm}$ while the radius of the beam on the surface was fixed. When $z_{f}=\infty$, the incident beam was collimated and unfocused. The sizes of the grid elements for the Monte Carlo simulations in the $x, y, z$ directions were $0.05 \mathrm{~cm}, 0.05 \mathrm{~cm}$, and 0.025 $\mathrm{cm}$, respectively. It can be seen that focusing had a dramatic effect on the absorption distribution in the turbid medium. As the depth of the focal point increased, the peak absorption density decreased, the focus became blurred, and the pattern of absorption expanded. The key parameter was the transport mean free path defined as $1 /\left[\mu_{a}+\mu_{s}(1-g)\right]$, which was $0.099 \mathrm{~cm}$. Therefore, the focusing had a significant effect when the depth of the focal point was less than or comparable with the transport mean free path.

When the indices of refraction between the ambient medium and the turbid medium were mismatched, $n_{a}=1.0$ and $n_{t}=1.4$. The depth of the focal point was $z_{f}=0.1 \mathrm{~cm}$ based on propagation in air. Two reasons affected the absorption distribution in the mismatched scenario. First, the beam was defocused by the mismatched interface between the ambient medium and the turbid medium. Second, the mismatched 
boundary increased the internal light reflection and reduced light reflection into the ambient medium. Therefore, more light was absorbed near the boundary, and the light absorption was more spread out.

The absorption distribution was simulated when the focusing was corrected such that the unscattered light rays would reach the same point at $z_{f}=0.1 \mathrm{~cm}$ based on propagation in the turbid medium. This focus correction for mismatched index of refraction could be accomplished by use of specially designed external focusing optics.

Figure 2 shows the one-dimensional absorption distributions along the vertical line through the center of the light beam. Figure 2(a) demonstrated the effect of the depth of the focal point $z_{f}$. As the depth of the focal point $z_{f}$ increased, the peak absorption decreased, and the depth of peak absorption increased initially but quickly reached a plateau. Even if the depth of the focal point $z_{f}$ increased beyond $0.1 \mathrm{~cm}$, the depth of peak absorption was less than $0.1 \mathrm{~cm}$, which was approximately the transport mean free path. Therefore, focusing the depth of peak absorption significantly more than one transport mean free path into a turbid medium would prove to be inefficient although the peak absorption with a focused beam was greater than the peak absorption with an unfocused beam.

Figure 2(b) shows that the uncorrected focusing with a mismatched boundary had the lowest peak absorption because of defocusing. The corrected focusing with a mismatched boundary had the highest peak absorption because of the increased internal reflection at the boundary. For many therapeutic applications of light, such as the port wine stain treatments, the ratio between the absorption at the boundary and the peak absorption inside the tissues is the most significant parameter because it determines how well the skin surface can be preserved while the internal lesions are treated. Therefore, we plotted the normalized absorption distributions in Fig. 2(c). The focusing into index-matched turbid medium had the lowest surface-to-peak ratio of absorption, which was nearly $50 \%$. The uncorrected focusing with a mismatched boundary had the highest surface-to-peak ratio of absorption, which was approximately $85 \%$, and had the broadest distribution. The corrected focusing with a mismatched boundary had a slightly lower surface-topeak ratio of absorption but had a much narrower distribution than the uncorrected counterpart .

The distributions of the absorption density in the skin model as shown in Fig. 1(b) were also simulated. The radius of the optical beam $\rho_{f}$ was fixed. Several scenerios were simulated: (a) Matched indices of refraction between the ambient medium and the turbid medium, and $z_{f}=\infty$ (unfocused). (b) Matched indices, and $z_{f}=0.015 \mathrm{~cm}$. (c) Mismatched indices, and $z_{f}=\infty$. (d) Mismatched indices, and $z_{f}=$ $0.015 \mathrm{~cm}$ without focus correction. (e) Mismatched indices, and $z_{f}=0.015 \mathrm{~cm}$ with focus correction. The $0.006-\mathrm{cm}$ diameter blood vessel was buried $0.015 \mathrm{~cm}$ deep. The radius of the optical beam $\rho_{f}$ was $0.075 \mathrm{~cm}$.

Figure 3(a) shows the one-dimensional distributions along the vertical line through the center of the optical beam. Additionally, the one-dimensional distributions of the absorption density were plotted for more deeply buried blood vessels under the matched boundary condition in Figs. 3(b) and (c). The sizes of the grid elements for the Monte Carlo simulations were $0.002 \mathrm{~cm}$ in all the $x, y, z$ directions. Since the blood was much more absorbing than the other components in the skin model, the absorption density in the blood vessel was greater than that in the epidermis and dermis even when the beam was not focused. Under both matched and mismatched boundary conditions, focusing into the shallow blood vessel dramatically increased the absorption density in the blood vessel even when the optical beam was defocused by the mismatched boundary. Note that the depth of the blood vessel for Fig. 3(a) was approximately one transport mean free path as calculated by $\mu_{t e}{ }^{\prime} d_{e}+\mu_{t d}{ }^{\prime} d_{d}$, where $\mu_{t e}{ }^{\prime}$ and $\mu_{t d}{ }^{\prime}$ were the sum of the absorption coefficient and the reduced scattering coefficient for the epidermis and dermis, respectively, $d_{e}$ was the thickness of the epidermis, and $d_{d}$ was the distance between the bottom of the epidermis and the center of the blood vessel. As the distance between the center of the cylinder and the surface of the turbid medium was doubled and quadrupled, the effect of focusing decreased dramatically [Figs. 3(b) and (c)]. This was in agreement with the studies on focusing light into homogeneous turbid media.

A short-pulsed laser is often used to reach the so-called "thermal confinement" condition for localized treatment, where the laser pulse width is much shorter than the thermal relaxation time of the medium being treated. In thermally confined treatments, the above simulated absorption probability density can be converted into the energy absorption density by multiplying it with the pulse energy of the laser. The absorbed optical energy is converted into heat and causes an abrupt temperature rise. The instantaneous temperature rise is proportional to the local absorption density. For example, by use of a focused beam with a matched boundary [Fig. 2(c)], the surface temperature rise would be only $\sim 50 \%$ of the peak temperature rise inside the turbid medium. For a buried highly absorbing blood vessel, the surface temperature rise 
would be much lower than the peak temperature rise in the vessel when the beam is properly focused into the blood vessel.

\section{SUMMARY and DISCUSSION}

Focusing light into a homogeneous turbid medium had a significant impact on the absorption distribution in the turbid medium when the distance of the focal point from the surface of the turbid medium was less than or comparable with the transport mean free path. Focusing increased the peak absorption and narrowed the absorption distribution significantly. As the depth of the focal point $z_{f}$ increased, the peak absorption decreased, and the depth of peak absorption increased initially but quickly reached a plateau that was less than the transport mean free path. Therefore, focusing the peak absorption significantly more than one transport mean free path into a turbid medium would prove to be inefficient although the peak absorption with a focused beam was greater than the peak absorption with an unfocused beam.

A mismatched boundary between the ambient medium and the turbid medium deteriorated the focusing effectiveness, increased the absorption near the boundary, lowered the peak absorption, and broadened the absorption distribution. The focus correction improved the surface-to-peak ratio of absorption slightly but narrowed the absorption distribution significantly.

Focusing into a shallowly buried absorbing object was very effective. But the effectiveness was reduced significantly when the depth of the buried object was increased to more than one transport mean free path.

This study provides some guidance for improving clinical treatments of superficial lesions such as port wine stains. If the depth of the lesions are measured with techniques such as optical coherence tomography or high-frequency ultrasonography, the laser beam can be focused toward the lesions such as the vessel network for improved localization of energy. Of course, focusing into wrong locations surrounding the lesions would cause adverse effects. One of the techniques that may assist the focus aiming is photo-acoustic imaging. ${ }^{16}$ If the power of the treatment laser is reduced to a diagnostic (non-therapeutic) level, the laser can be used as the optical source for photo-acoustic imaging. The photo-acoustic signal is used to optimize the focusing such that an maximal amount of energy is absorbed by the lesions compared with the surrounding normal tissue. Once the focusing is completed, the laser power can be restored to the therapeutic level for treatments.

\section{ACKNOWLEDGMENTS}

This project was sponsored in part by the National Institutes of Health grants R29 CA68562 and R01 CA71980, and the National Science Foundation grant BES-9734491. 


\section{FIGURES}

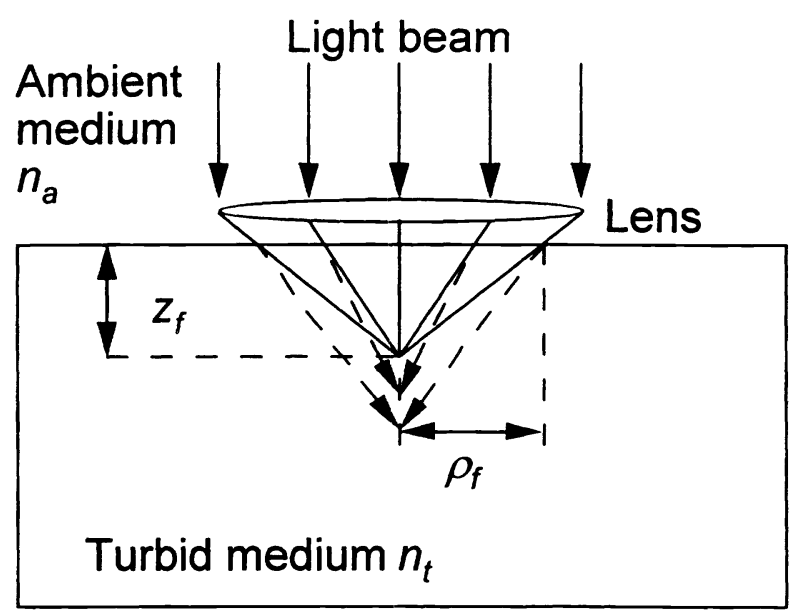

(a)

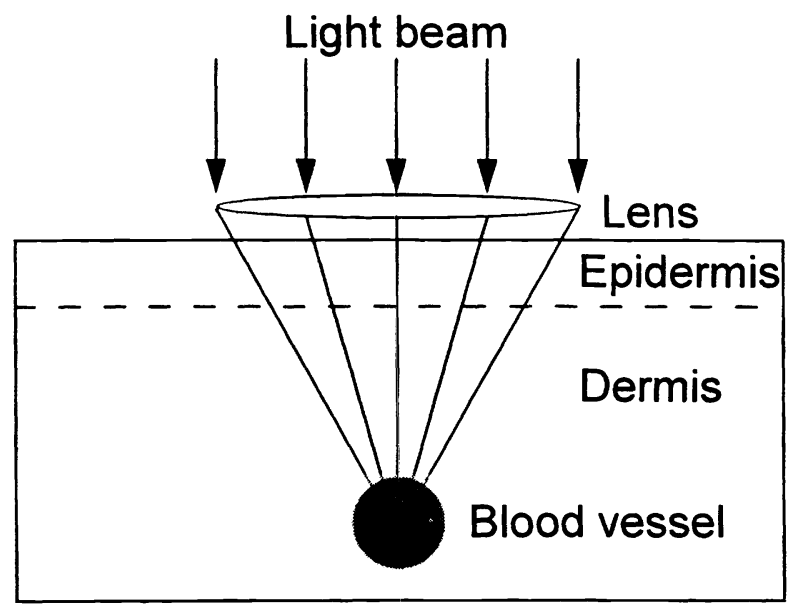

(b)

Fig. 1. (a) Schematic of focusing an optical beam into a turbid medium, where $z_{f}$ was the depth of the focal point in the turbid medium, $\rho_{f}$ was the radius of the optical beam on the surface of the turbid medium, $n_{a}$ was the index of refraction of the ambient medium, and $n$, was the index of refraction of the turbid medium. Focusing is illustrated for both matched boundary condition (solid converging lines) and mismatched boundary condition (dashed lines with arrows). (b) Schematic of a skin model containing the epidermal and dermal layers. A $0.006-\mathrm{cm}(60-\mu \mathrm{m})$ diameter blood vessel was buried $0.015 \mathrm{~cm}$ deep in the dermis unless stated otherwise. The thickness of the epidermis was $0.006 \mathrm{~cm}(60 \mu \mathrm{m})$. 


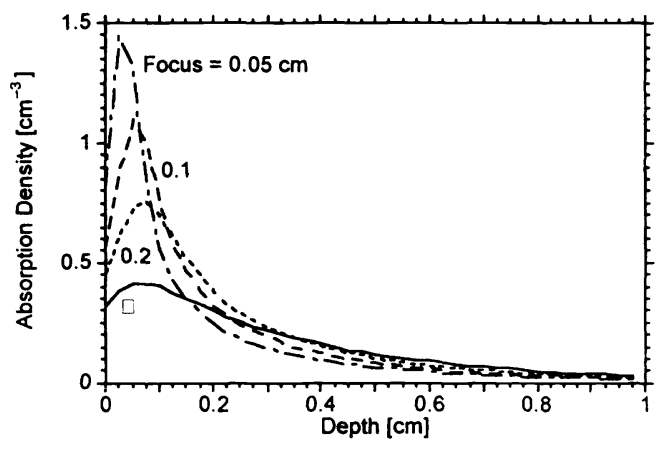

(a)

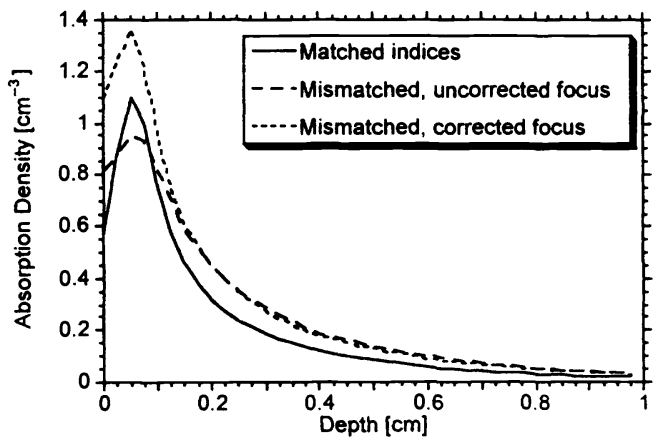

(b)

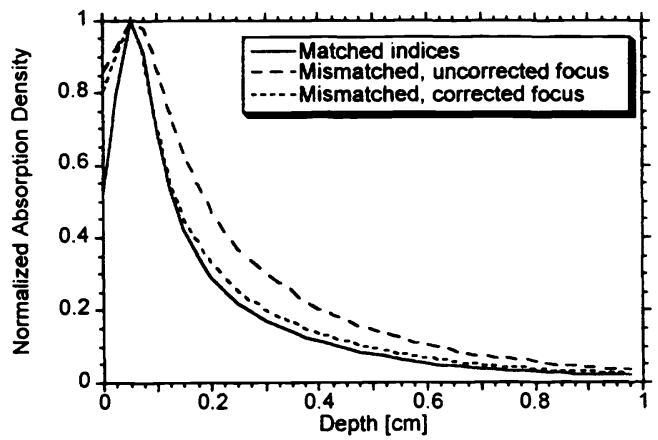

(c)

Fig. 2. One-dimensional depth distributions of the absorption density in the turbid medium under various conditions [see Fig. 1(a)]. (a) Comparison in the case of matched indices of refraction between the ambient medium and the turbid medium. (b) Comparison among the absorption density curves showing the effects of mismatched indices and focus correction. (c) Comparison among the normalized absorption density curves showing the effects of mismatched indices and focus correction. 


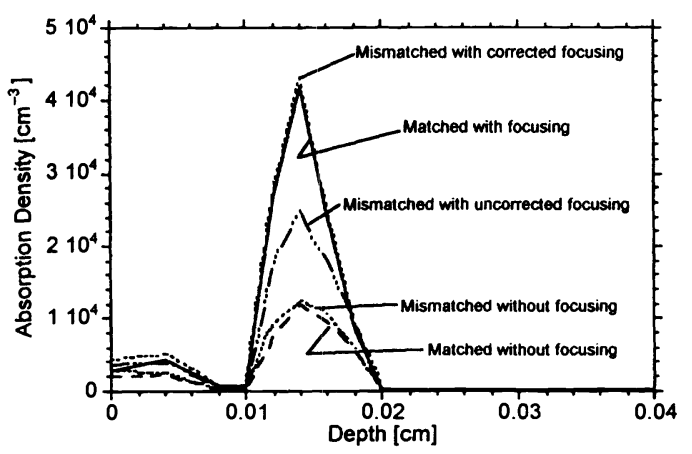

(a)

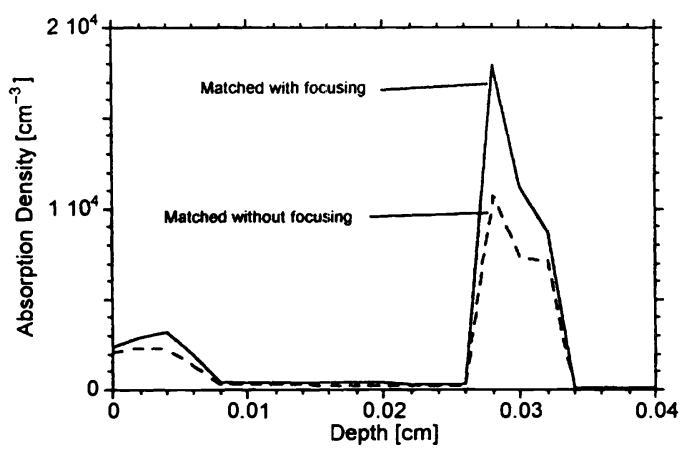

(b)

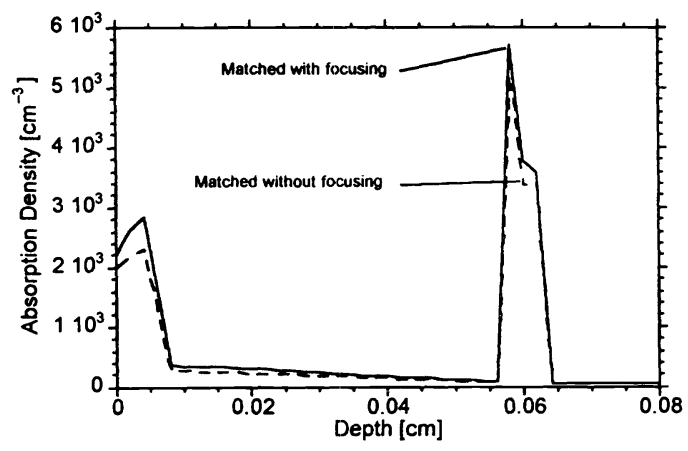

(c)

Fig. 3. One-dimensional depth distributions of the absorption density in the skin model under various conditions [see Fig. 1(b)]. (a) Both the effects of matched indices of refraction and focusing were considered. The distance between the center of the cylinder and the surface of the turbid medium was 0.015 $\mathrm{cm}$. (b) The effect of focusing under matched boundary condition was studied when the distance between the center of the cylinder and the surface of the turbid medium was $0.030 \mathrm{~cm}$. (c) The effect of focusing under matched boundary condition was studied when the distance between the center of the cylinder and the surface of the turbid medium was $0.060 \mathrm{~cm}$. 


\section{REFERENCES}

1. A. H. Hielscher, R. E. Alcouffe, and R. L. Barbour, "Comparison of finite-difference transport and diffusion calculations for photon migration in homogeneous and heterogeneous tissues," Phys. Med. \& Biol. 43, 12851302 (1998).

2. M. Schweiger and S. R. Arridge, "The finite-element method for the propagation of light in scattering media frequency domain case," Med. Phys. 24, 895-902 (1997).

3. A. H. Gandjbakhche, G. H. Weiss, R. F. Bonner, and R. Nossal, "Photon path-length distributions for transmission through optically turbid slabs," Phys. Rev. E 48, 810-818 (1993).

4. L. T. Perelman, J. Wu, I. Itzkan, and M. S. Feld, "Photon migration in turbid media using path integrals," Phys. Rev. Lett. 72, 1341-1344 (1994).

5. A. Singh and K. P. Gopinathan, "Confocal microscopy - a powerful technique for biological research," Current Science 74, 841-851 (1998).

6. B. C. Wilson and G. A. Adam, "Monte Carlo model for the absorption and flux distributions of light in tissue," Med. Phys. 10, 824-830 (1983).

7. S. T. Flock, B. C. Wilson, D. R. Wyman, and M. S. Patterson, "Monte-Carlo modeling of light-propagation in highly scattering tissues I: model predictions and comparison with diffusion-theory," IEEE Trans. Biomed. Eng. 36, 1162-1168 (1989).

8. S. A. Prahl, M. Keijzer, S. L. Jacques, and A. J. Welch, "A Monte Carlo model of light propagation in tissue," in Dosimetry of Laser Radiation in Medicine and Biology, G. J. Muller and D. H. Sliney, eds., Proc. Soc. Photo-Opt. Instrum. Eng. IS 5, 102-111 (1989).

9. S. L. Jacques and L.-H. Wang, "Monte Carlo modeling of light transport in tissues," in Optical Thermal Response of Laser Irradiated Tissue, A. J. Welch and M. J. C. van Gemert, eds., (Plenum Press, New York, 1995), pp. 73-100.

10. L.-H. Wang, S. L. Jacques, and L.-Q. Zheng, "MCML - Monte Carlo modeling of photon transport in multilayered tissues," Comp. Meth. and Prog. in Biomed. 47, 131-146 (1995). The MCML/CONV software package may be downloaded from URL: http://people.tamu.edu/ lwang.

11. A. Sassaroli, C. Blumetti, F. Martelli, L. Alianelli, D. Contini, A. Ismaelli, and G. Zaccanti, "Monte Carlo procedure for investigating light propagation and imaging of highly scattering media," Appl. Opt. 37, 7392 7400 (1997).

12. E. Okada, M. Firbank, M. Schweiger, S. R. Arridge, M. Cope, and D. T. Delpy, "Theoretical and experimental investigation of near-infrared light propagation in a model of the adult head," Appl. Opt. 36, 21 - 31 (1997).

13. L.-H. Wang, S. L. Jacques, and L.-Q. Zheng, "CONV - Convolution for responses to a finite diameter photon beam incident on multi-layered tissues," Comp. Meth. and Prog. in Biomed. 54, 141-150 (1997).

14. I. Lux and L. Koblinger, Monte Carlo Particle Transport Methods: Neutron and Photon Calculations (CRC Press, Boca Raton, 1991).

15. C. Sturesson and S. Andersson-Engels, "Mathematical modeling of dynamic cooling and pre-heating, used to increase the depth of selective damage to blood vessels in laser treatment of port wine stains," Phys. Med. Biol. 41, 413-428 (1996).

16. C. G. A. Hoelen, F. F. M. Demul, R. Pongers, and A. Dekker, "Three-dimensional photoacoustic imaging of blood vessels in tissue," Opt. Lett. 23, 648-650 (1998). 